\title{
Proceso comunicacional de manejo de residuos sólidos en negocios de alimentos y servicios. Caso de estudio restaurante de la Escuela de Gastronomía
}

\author{
Communicational process of solid waste management in food and service \\ businesses. Case study Gastronomy School restaurant
}

Iraida Maritza Gavilanez Alvarez. ${ }^{1}$, \& Susana Monserrat Zurita Polo. ${ }^{2}$, Recibido: 10-02-2020 / Revisado: 15-03-2020 /Aceptado: 04-04-2020/ Publicado: 06-05-2020

\begin{abstract}
.
DOI: https://doi.org/10.33262/concienciadigital.v3i2.1.1218

The processes that are developed in the gastronomic field in the Restaurant of the ESPOCH Gastronomy Career are carried out efficiently, so it is pertinent to make the polytechnic community known and socialize the general public through different communication processes as performs solid waste management which will improve the service provided by students and teachers in the environment in the aforementioned restaurant. It is important to disseminate how practices are carried out in terms of solid waste management, identifying the need to implement adequate environmental plans that facilitate and improve restaurant services, in this way both environmental and visual impacts can be greatly reduced. that could be generated by the accumulation of garbage. To carry out this research, a diagnosis was first made and then a baseline survey on the management of both organic and inorganic solid waste resulting from the process generated in the kitchen, and the processes that are developed in terms of recycling were identified. Within the course, through some research techniques such as observation, application of surveys, and interviews with both the responsible staff and users of the ESPOCH School of Gastronomy restaurant, Additionally, an environmental file was used to identify the characterization of areas of influence in both the physical, biotic, socio-cultural, and educational areas, among
\end{abstract}

\footnotetext{
1 Escuela Superior Politécnica de Chimborazo, Facultad de Recursos Naturales. Riobamba, Ecuador, igavilanez@espoch.edu.ec

2 Escuela Superior Politécnica de Chimborazo, Facultad de Recursos Naturales. Riobamba, Ecuador, susana.zurita@espoch.edu.ec
} 
ISSN: 2600-5859

Vol. 3, N².1, p. 25-40, mayo, 2020

others. The Environmental Standards TULAS BOOK VI Annex 6 for the Management of Non-Hazardous Solid Waste have also been consulted, and the Per Capita Production of waste that the restaurant generates has been determined, being $0.20 \mathrm{Kg} / \mathrm{Room} /$ day. o $0.45 \mathrm{lb} / \mathrm{Hab} /$ day and a total of waste generated per week of $113.91 \mathrm{Kg}$. or $250.61 \mathrm{lb}$ corresponding to a common week of work in the restaurant.

Keywords: Solid waste, Environmental Record, Pollution, Waste, Non-Hazardous Waste, Diffusion, Training

\section{Resumen.}

Los procesos que se desarrollan en el ámbito gastronómico en el Restaurante de la Carrera de Gastronomía de la ESPOCH son llevados de manera eficiente, por ello es pertinente dar a conocer a la comunidad politécnica y socializar a la ciudadanía en general mediante distintos procesos comunicacionales como se realiza el manejo de residuos sólidos lo cual permitirá mejorar el servicio brindado por estudiantes y docentes en el entorno en el mencionado restaurante. Es importante difundir cómo se realizan las prácticas en cuanto al manejo de residuos sólidos, identificando la necesidad de implementar planes ambientales adecuados que faciliten y mejoren los servicios en el restaurante, de esta manera se podrán reducir en gran medida los impactos tanto ambientales, como visuales que se pudieran generar por la acumulación de basura. Para la realización de la presente investigación se realizó primeramente un diagnóstico y luego un levantamiento de la línea base sobre el manejo de residuos sólidos tanto orgánicos como inorgánicos resultantes del proceso generado en la cocina, además se identificaron los procesos que se desarrollan en cuanto a reciclaje dentro de la carrera, a través de algunas técnicas de investigación como son la observación, aplicación de encuestas y entrevistas tanto al personal responsable como a usuarios del restaurante de la Escuela de Gastronomía de la ESPOCH. Adicionalmente se utilizó una ficha ambiental para identificar la caracterización de áreas de influencia tanto en el ámbito físico, biótico, socio-cultural, educativo entre otros, también se han consultado las Normas Ambientales TULAS LIBRO VI Anexo 6 para el Manejo de Desechos Sólidos no Peligrosos, y se ha determinado la Producción Per Cápita de residuos que el restaurante genera, siendo de $0.20 \mathrm{Kg} / \mathrm{Hab} /$ día. o $0.45 \mathrm{lb} / \mathrm{Hab} /$ día y un total de residuos generados por semana de $113.91 \mathrm{Kg}$. o $250.61 \mathrm{lb}$ correspondiente a una semana común de trabajo en el restaurante.

Palabras claves: Residuos sólidos, Ficha Ambiental, Contaminación, Desechos, Residuos no Peligrosos, Difusión, Capacitación 


\section{Introducción.}

En la actualidad se identifica diariamente a través de redes sociales, noticias, imágenes y videos la cantidad de basura que la humanidad genera, lo que está provocando un deterioro y altos índices de contaminación y afectación al medio ambiente, sin embargo, se debe considerar que no todos estos residuos son dañinos para el hombre, aparecen los denominados "residuos no peligrosos", los mismos que se encuentran catalogados así, por no presentar características de peligrosidad.

Se considera a los desechos sólidos comerciales dentro de los Desechos no Peligrosos, por cuanto son desechos generados por establecimientos comerciales y negocios restauranteros como hoteles, restaurantes, cafeterías, entre otros, su concepto está estipulado en las Normas Ambientales TULAS LIBRO VI Anexo 6 para el Manejo de Desechos Sólidos no Peligrosos desde su generación hasta su disposición final. (ESPOL LIBRO VI, 2012)

La generación de desechos puede considerarse un problema para la sociedad, si no se realiza una correcta clasificación de los residuos generados en áreas de cocina de los restaurantes, si bien las actividades humanas modernas, el consumismo, el facilismo y la falta de conciencia ambiental han incrementado la cantidad de basura generada, también es cierto que el daño al ambiente como afecciones a la salud está latente, esta situación puede cambiar si tanto responsables como usuarios del restaurante colaboran mancomunadamente.

Para Evaluar dicho problema se consideró la aplicación de una ficha ambiental cuya finalidad fue establecer aquellos parámetros que han sido afectados basados en acciones que provocan contaminación o que generen alteraciones al ambiente, obteniéndose información importante y pertinente de la Dirección de Escuela, además se aplicaron encuestas a personas encargadas de la limpieza y aseo del restaurante, incluyendo además a un grupo de estudiantes, se efectuó el reconocimiento del sitio de preparación de los distintos menús que se elaboran actualmente en el restaurante. (Gómez Orea, 2007)

Para realizar el levantamiento de la línea base se procedió a recopilar datos e formación importante mediante entrevistas con el Director y secretaria de la escuela de Gastronomía, visita in situ al local para ser partícipes de las diferentes preparaciones en calidad de participantes politécnicos, se observó cómo se lleva a cabo el manejo de residuos sólidos no peligrosos, se utilizó como parámetro importante la aplicación de una ficha ambiental, jerarquizando los tipos de impactos positivos y negativos, se generó la clasificación de forma adecuada tanto de los residuos sólidos orgánicos como de los inorgánicos además se los clasificó como reciclables y no reciclables, se procedió a establecer la producción precipita PPC diaria de los residuos generados en el área determinada y la cantidad semanal de Residuos sólidos producidos.

El objetivo de este proceso ambiental es dar a conocer los resultados del manejo adecuado de los residuos sólidos, planteando soluciones viables que permitan el correcto 
aprovechamiento y reciclaje de aquellos componentes comercializables considerados por algunos como basura.

\section{Metodología.}

La presente investigación tuvo como escenario principal el área de cocina del restaurante de la carrera de Gastronomía de la ESPOCH, lugar donde se realiza la preparación y procesamiento de alimentos, y por ende donde se genera gran cantidad de residuos sólidos RS, mediante el presente estudio se pretende establecer la composición física de los mismos, cantidad de residuos sólidos generados por semana y la producción per cápita de estos. Se aplicó una ficha ambiental para evaluar el impacto ambiental del restaurante, a través de parámetros de afectación considerando contaminantes o generadores de alteraciones al ambiente; se utilizaron técnicas de investigación como observación para reconocimiento del sitio estableciendo los productos que se utilizan a diario para la elaboración de los distintos alimentos, la cantidad de residuos sólidos generados y las posibles fuentes de contaminación, entrevistas a los responsables del restaurante y encargadas de realizar la limpieza, el aseo y preparación de alimentos, así como encuestas a los usuarios y consumidores en el restaurante, para realizar el levantamiento de la línea base se generó una investigación de campo.

\section{Los métodos utilizados son los siguientes:}

Método Científico: Siguiendo un conjunto de pasos en la investigación desde la observación hasta la determinación de resultados a través de la generación de un Plan de Manejo Ambiental.

Método Cuantitativo: Por cuanto se obtienen datos que serán procesados e interpretados para determinar la cantidad de residuos sólidos generados y la Producción Per Cápita de basura en el restaurante de la carrera de Gastronomía.

La investigación es de tipo cuasi-experimental y de campo porque se basa en la clasificación del tipo de residuos sólidos en la cocina del restaurante y su estudio para determinar los residuos sólidos que se generan en mayor proporción y establecer propuestas claras y definidas para su mejor procesamiento.

\section{Análisis de desechos (basura).}

\section{Obtención de información.}

Luego de que se ha establecido el tamaño muestral (puntos a muestrear) mediante muestreo sistemático se procedió a trabajar de la siguiente manera:

- Selección del sitio para la caracterización y clasificación de los distintos residuos.

- Observación e identificación de residuos sólidos generados en el restaurante. 
- Diálogo con las autoridades acerca del trabajo a realizarse, estableciendo su importancia, y solicitud de información para el estudio a través del muestreo en el restaurante.

- Difusión de objetivos y metodología del trabajo a desarrollarse.

- Socialización sobre la forma de realizar el muestreo durante 4 días de la semana en el lapso de 2 semanas consecutivas.

- Provisión de fundas plásticas vacías (específicas por colores) a los participantes con la socialización de las instrucciones acerca de la forma como se va a proceder con la recolección y clasificación de los residuos tanto orgánicos (fundas verdes), como inorgánicos (fundas negras) y fundas pequeñas para la basura del baño. Para el efecto las fundas fueron codificadas y rotuladas de manera adecuada mediante un código numerado para evitar confusiones y posteriores errores.

- Conjuntamente se recolectaron fundas plásticas con los residuos clasificados para realizar el respectivo pesaje.

- Finalmente se procedió a realzar la caracterización respectiva y procedimiento restante.

\section{Objetivo General}

Identificar el manejo que se da a los residuos sólidos en el restaurante de la Carrera de Gastronomía de la ESPOCH y difusión de dichos procesos mediante procesos comunicacionales sencillos para motivar el cuidado y preservación del medio ambiente.

\section{Objetivos Específicos}

- Definir procedimientos sencillos y prácticos para un manejo efectivo de los residuos sólidos en el restaurante de la carrera de Gastronomía

- Difundir los planes de manejo de residuos sólidos y promover mejor comportamiento ambiental por parte de responsables y usuarios del restaurante.

- Incentivar un manejo adecuado e integral de los residuos sólidos a través de un sistema que incluya la clasificación adecuada dentro del restaurante mediante una diferenciada recolección, tratamiento y posterior disposición final.

\section{Resultados.}

\section{Residuos sólidos.}

Un residuo se define a aquella materia que no posee algún valor económico, o a cualquier material que su propietario decida destinarlo al abandono, por lo que resulta muy apropiado denominarlo como residuo por las características que presentan como desperdicios, desechos o basuras, debido a que implica un deseo/necesidad de eliminar dicho materiales, debido a que no se les atribuye un valor suficiente para conservarlos. Esta carencia de valor puede deberse a varias causas:

No poder reutilizar los materiales abandonados por no existir la tecnología adecuada de recuperación. La dificultad de comercialización de los productos recuperados, debido a los 
elevados costos de recuperación; o la no existencia de mercados para estas materias, o a rechazo de los productos. (Bustos, 2007)

Un residuo sólido es aquel objeto, sustancia o elemento que se lo puede reutilizar o reciclar mediante sistemas de tratamiento ya sea de forma manual para los residuos orgánicos o mediante el uso de máquinas recicladoras para los residuos inorgánicos.

Clasificación por las características de os residuos asociadas al manejo de los residuos:
a. Residuos sólidos orgánicos
b. Residuos sólidos inorgánicos
c. Desechos sólidos generales
d. Desechos sólidos pétreos.
e. Desechos peligrosos.

\section{Generación de residuos.}

\section{Producción Per Cápita PPC}

La producción de los residuos sólidos domésticos constituye una variable que depende del tamaño de la población y las características socioeconómicas, la misma que asocia tamaño de la población, cantidad de residuos y el tiempo expresándose en $\mathrm{Kg} /$ habitante/día.

Teóricamente se la puede estimar haciendo uso de la siguiente fórmula: (Bustos, 2007)

\section{Manejo de residuos sólidos.}

Constituyen el conjunto de todas aquellas políticas y procedimientos que conforman el sistema de manejo de los residuos sólidos y cuya finalidad es realizar una gestión que sea económica y ambientalmente adecuada. (Avellaneda, 2000)

\section{Sistema de manejo de residuos sólidos}

Este importante determinar que el sistema de manejo de residuos sólidos se compone de cinco subsistemas que son: (Muñoz, 2008)

Fig. No 1 sistema de manejo de residuos sólidos (Canter, 1997)

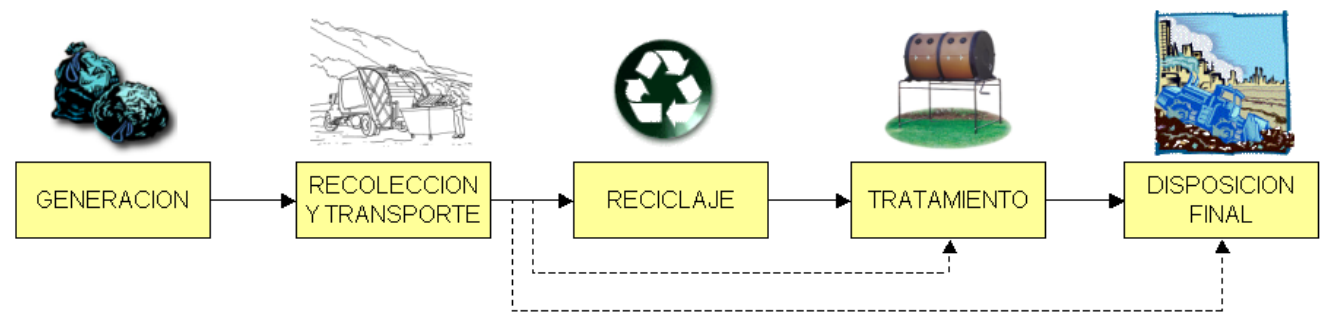




\section{Impacto ambiental.}

Es cualquier alteración de las condiciones ambientales o la creación de nuevas condiciones ambientales beneficiosas (+) o adversas (-) causadas por una acción de una obra de desarrollo. (Canter, 1997)

\section{Evaluación de impacto ambiental. EIA}

Está definida como la identificación y valoración de los impactos potenciales de proyectos, planes, programas o acciones normativas relativas a los componentes físico-químicos, bióticos, culturales y socioeconómicos del entorno.

\section{Plan de Manejo Ambiental de Residuos Sólidos - Base Legal}

Todas las actividades productivas, están regidas en la Legislación Ecuatoriana vigente, por tanto, bajo los lineamientos técnicos y la normativa respecto a la preservación del medio ambiente, se ha considerado para la realización del presente estudio ha sido analizada en el marco de los instrumentos jurídicos descritos a continuación:

La constitución política de la república del Ecuador, publicada en el R.O. No 449 del 20 de octubre del 2008 Título II: DERECHOS, Capítulo segundo: Derechos del buen vivir, Sección segunda: Ambiente sano, en los artículos 14, 15 y 66 numeral 27.

\section{Normativa Ambiental}

Según la normativa nacional Texto Unificado de Legislación Ambiental Secundaria TULASLibro VI: Anexo 6, Literal 4 y el Anexo 2. Esta norma establece los procedimientos generales en el manejo de los desechos sólidos no peligrosos, desde la generación hasta la disposición final; y las normas de calidad que deben cumplir los desechos sólidos no peligrosos para cumplir con estándares que permitan la preservación del ambiente.

El Plan de Manejo Ambiental. PMA Es un documento que establece en detalle y en orden cronológico las acciones que se requieren para prevenir, mitigar, minimizar, controlar, corregir y compensar los posibles impactos ambientales negativos, o acentuar los impactos positivos causados en el desarrollo de una acción propuesta. Por lo general el PMA consiste en varios sub-planes o programas, que dependen de las características de la actividad o proyecto propuesto. (Gavilánez, 2012)

\section{Resultados Esperados.}

- Mediante una adecuada difusión sobre el manejo de residuos sólidos se espera que los usuarios generen conciencia y comportamiento ambiental, apoyando procesos de reducción, reciclaje y reutilización de los residuos sólidos. 
ISSN: 2600-5859

Vol. 3, N².1, p. 25-40, mayo, 2020

- Con conocimiento sobre el manejo de residuos sólidos se espera que los encargados del restaurante realicen de forma habitual la clasificación de los residuos sólidos en la fuente, lo que disminuirá los impactos ambientales en la carrera de Gastronomía.

El presente Plan de Manejo ambiental consta de:

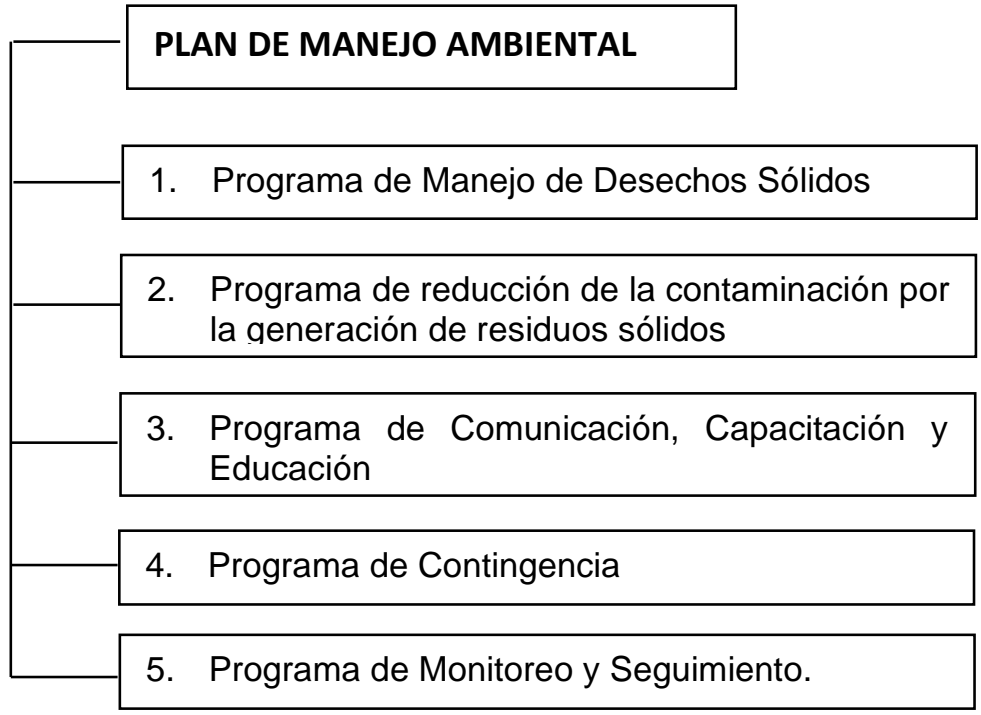

Fuente: Gavilanez, M., Zurita, M., Moreno A.

\section{Programa de Manejo de Desechos Solidos}

El Plan de Manejo de Desechos Sólidos (PMD) ha sido creado para definir procedimientos como son: clasificar en la fuente, almacenar correctamente, reutilizar, reciclar y disponer adecuadamente los desechos sólidos generados en el restaurante de la Escuela de Gastronomía de la ESPOCH. para el cual se recomienda el manejo adecuado, además de un plan de minimización de desechos, el control y las medidas de prevención, tratamiento y disposición final para no causar contaminación en los recursos agua, aire y suelo. (Gavilánez, 2012)

\section{Clasificación de los desechos sólidos}

Se han identificado los siguientes tipos de desechos sólidos:

- Orgánicos

- Inorgánicos

Por ser residuos incompatibles, su manejo debe ser por separado, así como se plantea en el presente programa. 
- Frecuencia de generación de desechos

- En la siguiente tabla se describe detalladamente los tiempos en los que se genera los desechos sólidos.

Tabla No 1 Frecuencia De Generación De Desechos

\begin{tabular}{lll}
\hline Tipo De Desechos & Semanal & Diario \\
\hline Desechos De Vidrio & $\mathrm{X}$ & \\
Desechos Plásticos & & $\mathrm{X}$ \\
Desechos Orgánicos & & $\mathrm{X}$ \\
Cartón Y Papel & & $\mathrm{X}$ \\
\hline
\end{tabular}

Fuente: Gavilanez, M., Zurita, M., Moreno A.

\section{Procedimientos de manejo de desechos sólidos}

\section{Impactos Para Prevenir}

- Los impactos para prevenir por medio del programa de manejo de desechos sólidos son:

- Impacto visual, mal aspecto en sus alrededores y parte interior, que no de una buena imagen del restaurante.

- Impacto en la salud, prevenir potenciales enfermedades por la presencia de vectores infectos contagiosos o transmisores de enfermedades.

\section{Manejo}

Para el manejo de desechos sólidos comunes se debe tomar en cuenta ciertas disposiciones generales:

Clasificación en la fuente: los desechos serán separados de acuerdo con su clase en la fuente generadora, para esto se deberá proveer de recipientes apropiados para cada uno de ellos identificados por color de acuerdo al tipo de desechos.

Color verde: para desechos orgánicos

Color negro: para desechos inorgánicos

\section{Medidas preventivas para la generación de desechos}

Las medidas preventivas para la generación de desechos consisten en la implementación de las 3R, es decir, reducir, reusar, reciclar en la medida de lo posible. Para ello se debe implementar el sistema de separación de desechos y de compactación del cartón, papel y plástico para disminuir su volumen, para de esta manera reducir al máximo los desechos y optimizar la disponibilidad de espacio en el centro de acopio. (Muñoz, 2008) 


\section{Medidas de disminución o minimización de desechos}

- La disminución de desechos puede ser una práctica de todos los días a ser implementada internamente, por medio de capacitación.

- La política de disminución de desechos debe estar dirigida a utilizar en todos los casos posibles envases, de cualquier tipo y finalidad, que sean retornables. Con esto se lograría no generar residuos.

Programa de reducción de la contaminación por la generación de residuos sólidos.

\section{Prevención de la contaminación}

El uso de materiales, procesos, o prácticas que reducen o eliminan la creación de contaminantes o basuras en la fuente de origen, todo ello previo a reciclaje, tratamiento o disposición. Incluye el diseño de productos y procesos que conducen a una educación o eliminación de la contaminación producida por el fabricante o usuario de los productos. (Gavilánez, 2012)

\section{Un programa efectivo:}

- Protege la salud pública y el medio ambiente

- Reduce el riesgo de responsabilidad civil o criminal

- Reduce los costos de operación

- Aumenta la moral y nivel de participación de los empleados

- Mejora la imagen de la compañía en la comunidad

\section{Programa de comunicación, capacitación y educación.}

El poder está en la información, quienes tienen la información adecuada preservan su mente y por ende crean hábitos y conductas en beneficio no solo personal si no principalmente colectiva, en este sentido es importante definir planes de comunicación, capacitación y educación dirigidos a los responsables y usuarios del restaurante de la carrera de Gastronomía. En este sentido es trascendental difundir el plan de manejo ambiental PMA, explicar su alcance y ejes de trabajo, a través del cual se generará mayor conciencia ambiental y cambio de actitud en beneficio de la sociedad en general.

La fase de socialización y difusión se la realiza a través del plan de capacitación, el mismo que será dirigido a los responsables del restaurante de la Escuela de Gastronomía de la ESPOCH en concordancia con los resultados obtenidos del diagnóstico realizado, el cual pretende fomentar un adecuado manejo de los residuos sólidos y un cambio de mentalidad en aquellas personas que dedican su labor a generar beneficio de las grandes sociedades.

La temática a tratarse en el proceso de capacitación incluye un módulo de gestión local de residuos sólidos priorizando los siguientes aspectos: 
- Socialización y difusión del estudio realizado.

- Generalidades y definiciones básicas de RS, origen y clasificación.

- Manejo adecuado de los residuos sólidos: realidad local.

- Uso de las 3R: reducción, reutilización y reciclaje.

\section{Programa de Rehabilitación de las Áreas Afectadas}

Establecer con las autoridades la implementación de mingas de limpieza y mantenimiento adecuado de las áreas que circundan al Restaurante en estudio con la finalidad de mitigar la contaminación visual, promoviendo un plan de recuperación, ornamentación y revegetación natural. Colocar señaléticas como rótulos y slogans con mensajes ambientales de manera que se identifique el área como un área importante de recuperación y la prohibición a botar basura fuera de los respectivos contenedores.

\section{Programa de Contingencia}

Este plan establece medidas de acción para enfrentar accidentes y cualquier situación de emergencia durante la operación de las actividades exploratorias, contempla:

- Determinación de medidas para la prevención de la contaminación.

- Planificación, ejecución, coordinación y control de la implementación de los procedimientos para la prevención de la contaminación.

- La centralización de la información y mejor utilización de los recursos disponibles.

- El empleo óptimo de los recursos, tanto humanos como materiales, para un rápido y adecuado control de la contaminación.

\section{Programa de Monitoreo y Seguimiento}

Con el propósito de monitorear y controlar el estado del área de estudio se recomienda realizar semestralmente una evaluación del manejo de residuos sólidos. En cuanto al componente social, es importante que una vez realizada la difusión del PMA y capacitaciones programadas en cuanto a concienciación y el fomento de la clasificación adecuada de desechos y residuos sólidos, se determine cuál es la percepción de los usuarios del

restaurante, así como los estudiantes y personal a cargo del mismo respecto a las mejorías que se evidencien luego del cumplimiento del presente Plan de Manejo Ambiental. (Gavilánez, 2012)

Luego de un año se recomienda actualizar el diagnóstico del sistema de aseo, recolección y tratamiento de los residuos sólidos con la finalidad de actualizar la producción per cápita de los residuos, tipo y características de los subproductos, estimación de porcentajes tanto de residuos orgánicos, como inorgánicos y efectiva evaluación del modelo de gestión vigente en coordinación con las autoridades de la escuela. 


\section{Materiales y métodos}

Caracterización del área de estudio

Provincia: Chimborazo

Cantón: Riobamba

Parroquia: Lizarzaburu

Ubicación: $\quad$ Panamericana Sur Km 1¹⁄2

Tabla $\mathbf{N}^{\circ} 2$ Materiales y Equipos

\begin{tabular}{|c|c|}
\hline Equipos y Materiales & $\begin{array}{l}\text { Botiquín de emergencia } \\
\text { Tijeras } \\
\text { Termómetro } \\
\text { Refrigeradores } \\
\text { Guantes } \\
\text { Fundas plásticas (verdes y negras) }\end{array}$ \\
\hline Materiales de Oficina & $\begin{array}{ll}\begin{array}{l}\text { Lápiz } \\
\text { Hojas de evaluación }\end{array} & \text { Marcadores } \\
\text { Computadora } & \text { Hojas de papel bond } \\
\text { Esferos } & \text { Ficha Ambiental } \\
\text { Fundas de polietileno (fundas para basura) de color negro domestica de 29" x 36" } \\
\text { o 74cm x 92cm (No existe norma INEN al respecto). } \\
\text { Fundas de polietileno (fundas para basura) de color verde domestica de 23" x 28" } \\
\text { o } 58 \mathrm{~cm} \text { x 71cm (No existe norma INEN al respecto). } \\
\text { Balanza de plato con capacidad mínima de } 30 \mathrm{Kg} \text {. } \\
\text { Cuaderno de apuntes, esferos. } \\
\text { Marcadores para rotular las fundas de muestreo. }\end{array}$ \\
\hline $\begin{array}{l}\text { Materiales para el Muestreo } \\
\text { y caracterización. }\end{array}$ & $\begin{array}{l}\text { Guantes de caucho. } \\
\text { Hojas para encuestas, etiquetas. } \\
\text { Mandil, mascarillas, agua, alcohol y desinfectante }\end{array}$ \\
\hline
\end{tabular}

Fuente: Gavilanez, M., Zurita, M., Moreno A.

\section{Resultados Y Discusión}

Luego de haber realizado la separación de los residuos sólidos

Tabla $\mathbf{N}^{\circ} 3$ Residuos Sólidos lbs y Kg (1 ${ }^{\text {a }}$. semana)

\begin{tabular}{|c|c|c|c|c|c|c|c|c|c|}
\hline \multicolumn{10}{|c|}{ Residuos sólidos generados en el Restaurante de la Escuela de Gastronomía } \\
\hline Día & $\begin{array}{l}\text { Materia. } \\
\text { Orgánica } \\
\text { (lb) }\end{array}$ & $\begin{array}{l}\text { Papel. } \\
\text { (lb) }\end{array}$ & $\begin{array}{l}\text { Cartón } \\
\text { (lb) }\end{array}$ & $\begin{array}{l}\text { Pl. S } \\
\text { (lb) }\end{array}$ & $\begin{array}{l}\text { Pl. R } \\
\text { (lb) }\end{array}$ & $\begin{array}{l}\text { Vidrio. } \\
\text { (lb) }\end{array}$ & $\begin{array}{l}\text { Metal } \\
\text { (lb) }\end{array}$ & $\begin{array}{l}\text { Materia } \\
\text { Inorgánica } \\
\text { (lb) }\end{array}$ & $\begin{array}{l}\text { Peso } \\
\text { total } \\
\text { lb/día }\end{array}$ \\
\hline Lunes & 56.30 & 1.54 & 0.24 & 0.26 & 0.98 & 0.19 & 0.00 & 3.21 & 59.51 \\
\hline Martes & 68.59 & 1.66 & 0.36 & 1.18 & 0.18 & 1.07 & 0.39 & 4.84 & 73.43 \\
\hline Miércoles & 58.60 & 1.46 & 0.23 & 0.28 & 0.97 & 0.21 & 0.00 & 3.15 & 61.75 \\
\hline Jueves & 62.59 & 1.32 & 0.64 & 1.46 & 0.24 & 1.54 & 0.00 & 5.20 & 67.79 \\
\hline Peso Semanal lb & 246.08 & 5.98 & 1.47 & 3.18 & 2.37 & 3.01 & 0.39 & 16.4 & 262.48 \\
\hline Promedio diario lb & 61.52 & 1.50 & 0.37 & 0.80 & 0.59 & 0.75 & 0.09 & 4.10 & 65.62 \\
\hline
\end{tabular}

FUENTE: Gavilanez, M., Zurita, M., Moreno A. 
ISSN: 2600-5859

Tabla $\mathbf{N}^{\circ} 4$ Residuos Sólidos lbs y Kg (2a . Semana)

\begin{tabular}{lllllllllll}
\hline \multicolumn{1}{l}{ Residuos sólidos generados en el Restaurante de la Escuela de Gastronomía } \\
\hline & $\begin{array}{l}\text { Materia } \\
\text { Orgánica } \\
\text { Día }\end{array}$ & $\begin{array}{l}\text { Papel } \\
(\mathbf{l b})\end{array}$ & $\begin{array}{l}\text { Cartón } \\
(\mathbf{l b})\end{array}$ & $\begin{array}{l}\text { PI. } \\
(\mathbf{l b})\end{array}$ & $\begin{array}{l}\text { Pl. R } \\
(\mathbf{l b})\end{array}$ & $\begin{array}{l}\text { Vidrio } \\
(\mathbf{l b})\end{array}$ & $\begin{array}{l}\text { Metal } \\
(\mathbf{l b})\end{array}$ & $\begin{array}{l}\text { Materia } \\
\text { Inorgánica } \\
\text { (lb) }\end{array}$ & $\begin{array}{l}\text { Peso } \\
\text { total } \\
\mathbf{l b / d i ́ a ~}\end{array}$ \\
Lunes & 50.27 & 1.35 & 0.65 & 0.56 & 0.78 & 0.34 & 0.14 & 3.82 & 54.09 \\
Martes & 63.44 & 1.23 & 0.72 & 0.97 & 0.24 & 0.67 & 0.45 & 4.28 & 67.72 \\
Miércoles & 53.69 & 1.99 & 0.38 & 0.45 & 0.67 & 0.27 & 0.30 & 4.06 & 57.75 \\
Jueves & 66.34 & 1.80 & 0.69 & 0.87 & 0.43 & 0.54 & 0.38 & 4.71 & 71.05 \\
$\begin{array}{l}\text { Peso Semanal lb } \\
\text { Peso Semanal Kg }\end{array}$ & 233.74 & 6.37 & 2.44 & 2.85 & 2.12 & 1.82 & 1.27 & 16.87 & $\mathbf{2 5 0 . 6 1}$ \\
Promedio diario Lb & 58.44 & 1.59 & 0.61 & 0.71 & 0.53 & 0.46 & 0.32 & 4.22 & $\mathbf{1 1 3 . 9 1}$ \\
\hline
\end{tabular}

Fuente: Gavilanez, M., Zurita, M., Moreno A.

Tabla $\mathbf{N}^{\circ} 5$ Producción Per Cápita De Residuos Sólidos

\begin{tabular}{|c|c|c|c|c|}
\hline Dias Muestreados & $\begin{array}{l}\text { Peso Total De } \\
\text { Los Residuos En } \\
\text { lb }\end{array}$ & $\begin{array}{l}\text { Población } \\
\text { Promedio } \\
\text { Días }\end{array}$ & $\begin{array}{l}\text { Ppc } \\
\text { lb/Persona/ } \\
\text { Día }\end{array}$ & $\begin{array}{l}\text { Ppc } \\
\text { lb/Persona/ } \\
\text { Día }\end{array}$ \\
\hline Lunes & 54.09 & 130 & 0.42 & 0.19 \\
\hline Martes & 67.72 & 145 & 0.47 & 0.21 \\
\hline Miércoles & 57.75 & 132 & 0.44 & 0.20 \\
\hline Jueves & 71.05 & 150 & 0.47 & 0.21 \\
\hline Peso Semanal lb & 250.61 & 557 & 0.45 & 0.81 \\
\hline Promedio diario lb & 62.65 & 139.25 & 0.45 & 0.20 \\
\hline
\end{tabular}

Fuente: Gavilanez, M., Zurita, M., Moreno A.

Luego del trabajo de campo en las dos semanas de recolección y separación de los residuos generados en el restaurante se han obtenido los siguientes resultados: una generación de residuos sólidos de $250.61 \mathrm{lb}$ o $113.91 \mathrm{Kg}$. por semana y una $\mathrm{PPC}$ de $0.20 \mathrm{Kg} / \mathrm{Hab} /$ día. o $0.45 \mathrm{lb} / \mathrm{Hab} /$ día que se encuentra dentro de los parámetros aceptables para esta determinación.

Los resultados que arrojaron las encuestas aplicadas establecieron que los estudiantes de quinto semestre A y B son quienes se encuentran a cargo del Manejo del servicio del restaurante, bajo la Dirección y Coordinación de dos Chefs docentes de la escuela; la recolección de los residuos se lo hacen en fundas plásticas en su mayoría, las mismas que cumplen con normas establecidas en cuanto a colores; se cuenta con un servicio público de recolección de basura luego que se ha depositado en los respectivos tachos institucionales, el recorrido realizado se lo efectúa a través de una camioneta aproximadamente 4 veces por semana durante la tarde conforme lo indican los encuestados. 
No existe información por parte de las autoridades acerca del cambio de los horarios de recolección.

El mayor porcentaje de los encuestados coinciden con que el servicio de recolección es bueno, respecto de un bajo porcentaje que piensan que es regular.

La mayoría de los encuestados no tienen claro conocimiento acerca del destino final de los desechos sólidos generados en la Facultad, sin embargo están conscientes del impacto ambiental que puede generar en su salud si no son tratados de manera debidamente.

En función de los resultados obtenidos en la encuesta realizada, se determina que el manejo de la basura en el restaurante es regular y que la disposición de basura la califican como adecuada en un $50 \%$, el $25 \%$ indica no conocer, el otro $25 \%$ lo consideran como inadecuado.

\section{Conclusiones.}

- El restaurante de la Escuela de Gastronomía requiere de una mayor cantidad de recipientes para la recolección pues se manejan pocos recipientes con fundas adecuadas del color específico para poder clasificar debidamente los residuos orgánicos tal y como debería ser para posterior reutilización.

- La generación de una clasificación adecuada podría permitir otra manera de utilización de los residuos para incluirlos en procesos culinarios optimizando por ejemplo la piel de las frutas y residuos de verduras permitirían hacer otro tipo de fondos además de los clásicos.

- Se ha determinado la producción Per Cápita y la cantidad de residuos sólidos que genera el restaurante estableciendo que se encuentran dentro de parámetros establecidos por las normas.

- Se ha generado una ficha ambiental y se han definido los programas ambientales para la minimización de los impactos ocasionados por la clasificación de los residuos sólidos.

- Se ha incentivado la participación de los estudiantes para que colaboren juntamente con las autoridades para mejorar la situación de la generación de los residuos sólidos producidos y así establecer políticas que permitan evitar problemas ambientales.

- La difusión del manejo adecuado de los residuos sólidos ha generado una conciencia ambiental en los usuarios, lo que promueve un mejor funcionamiento del restaurante.

\section{Referencias bibliográficas.}

Avellaneda, A. (2000). Gestión Ambiental y Planificación del Desarrollo. Colombia: ECOE Ediciones.

Bustos, F. (2007). Manual de Gestión y Control Ambiental. Quito: Industria Gráfica.

Canter, L. (1997). Manual de Evaluación de Impacto Ambiental. Buenos Aires.

Espol Libro VI. (2012). Norma de calidad ambiental para el manejo y disposición de desechos nólidos no Obtenido de 
ISSN: 2600-5859

Vol. 3, N².1, p. 25-40, mayo, 2020

http://www.dspace.espol.edu.ec/bitstream/123456789/6078/55/LIBRO\%20VI\%20A nexo\%206\%20MAnejo\%20desechos\%20solido\%20no\%20peligrosos.pdf

Gavilánez, M. (07 de 2012). ESPOCH. Obtenido de Repositorio Institucional de la Escuela Superior Politécnica de

Chimborazo: http://dspace.espoch.edu.ec/handle/123456789/4313

Gómez Orea, D. (2007). Evaluación del Impato Ambiental. Madrid: Agrícola Española S.A.

Muñoz, P. (2008). Manual de Manejo de Residuos Sólidos Urbanos. Quito: Industria Gráfica.

NORMAS TECNICAS

Ecuador. Ministerio Del Ambiente.- Texto Unificado de Legislación Secundaria (TULAS).Quito: MAE, Libro VI, Anexos I.II. VI.

Ley De Gestion Ambiental

Pérez Porto, J., \& Gardey, A. (2014). Obtenido de Definición de:: https://definicion.de/redsocial/ 
Vol. 3, N².1, p. 25-40, mayo, 2020

\section{PARA CITAR EL ARTÍCULO INDEXADO.}

Gavilanez Alvarez, I. M., \& Zurita Polo, S. M. (2020). Proceso comunicacional de manejo de residuos sólidos en negocios de alimentos y servicios. Caso de estudio restaurante de la Escuela de $\begin{array}{llll}\text { Gastronomía } \quad \text {. ConcienciaDigital, 25-40. } & \text { 3(2.1), }\end{array}$

https://doi.org/10.33262/concienciadigital.v3i2.1.1218

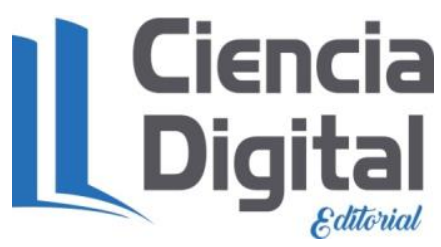

El artículo que se publica es de exclusiva responsabilidad de los autores y no necesariamente reflejan el pensamiento de la Revista Conciencia Digital.

El artículo queda en propiedad de la revista y, por tanto, su publicación parcial y/o total en otro medio tiene que ser autorizado por el director de la Revista Conciencia Digital.

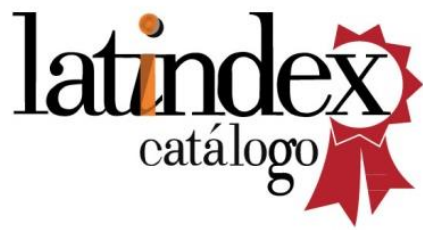

\section{Conciencia}

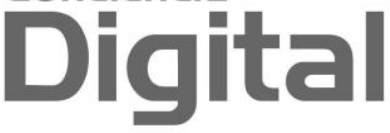

